\title{
Genetic expression and mutation profile analysis in different pathologic stages of hepatocellular carcinoma patients
}

\section{Xingjie Gao}

Tianjin Medical University

Chunyan Zhao

Tianjin Medical University

\section{Xiaoteng Cui}

Tianjin Medical University

Nan Zhang

Tianjin Medical University

\section{Yuanyuan Ren}

Tianjin Medical University

\section{Chao Su}

Tianjin Medical University

\section{Shaoyuan Wu}

Tianjin Medical University

\section{Zhi Yao}

Tianjin Medical University

Jie Yang ( $\nabla$ yangj@tmu.eud.cn )

Tianjin Medical University https://orcid.org/0000-0002-9669-1832

\section{Primary research}

Keywords: Expression, mutation, HCC, TCGA, pathologic stage

Posted Date: September 9th, 2020

DOI: https://doi.org/10.21203/rs.3.rs-71784/v1

License: (c) (i) This work is licensed under a Creative Commons Attribution 4.0 International License. Read Full License 


\section{Abstract}

Background: The expression and mutation of multiple genes are involved in the complicated mechanism regarding the occurrence and development of hepatocellular carcinoma (HCC). The clinical pathological stage of HCC is closely linked to clinical prognosis of liver cancer. This study aims at analyzing the gene expression and mutation profile of different clinical pathological stages of HCC (stage I, II, III-IV), based on 367 HCC cases included in TCGA cohort.

Results: We identified a series of targeting genes with copy number variation (CNV), which is statistically associated with gene expression. For instance, compared withthe normal group, CCNE2 gene is highly expressed in the tumor group and specificstage I group, which are associated withthree CNV types of single deletion, single gain, and amplification mutations. Protein interaction network construction and followed "Molecular Complex Detection" analysis indicated that the high expression of some cell cyclerelated genes in HCC, such as TTK, CDC20, ASPM, is positively correlated with CNV. Non-synonymous mutations mainly existed in some genes, such as TTN, TP53, CTNNB1, MUC16, andALB, however, we did not observe the association between thegene mutation frequency and the clinical pathological grade distribution. The rs121913396 and rs121913400 polymorphisms withintheCTNNB1 gene were associated with the high expression of CTNNB1 protein, but not linked to the clinical prognosis of HCC. We performed the random forest and decision tree approachesfor the modeling analysis and identified a group of genes related to different HCC pathological grades, such as the lowly expressed VIPR1, FAM99A, and GNA14 genes, or highly expressed CEP55, SEMA3F, and PRR11. Moreover, we conducted a principal component analysis (PCA) to obtain several genes associated with different pathological grades, including SLC27A5, ADAM17, SNRPA, SNRPD2, and ALDH2. Finally, we confirmed the highly expressed GAS2L3, SNRPA, SNRPD2 genes in the HCC tissues, for the first time, through a Chinese HLivH060PG02 cohort analysis.

Conclusions: The identification of the targeting genes, including GAS2L3, SNRPA, SNRPD2, provides insight into the molecular mechanisms associated with different prognosis of HCC.

\section{Background}

Hepatocellular carcinoma (HCC) is the primary histological subtype of liver cancer [1-3]. A series of factors, including the genetic, epigenetic changes, chronic hepatitis $B / C$ virus infection, aflatoxin exposure, smoking, obesity, and diabetes, contribute to the progression, diagnosis, and prognosis of HCC [4-6]. The clinical pathological stage of HCC is closely linked to clinical prognosis of liver cancer $[4,5]$. For the HCC cases with early pathological stage, the radical therapies (e.g., resection, radiofrequency ablation, transplantation, et al.) are valid and feasible [1]. It is thus meaningful to identify the potential genes, which is associated with the pathological stage I, II, III-IV of HCC. 
The Cancer Genome Atlas (TCGA), a public database, provides the multiple-genomics data from more than thirteen types of cancer, including gene expression, copy number variation (CNV), simple nucleotide variation (SNV), single nucleotide polymorphism (SNP), DNA methylation, and clinical information, etc. [7]. TCGA cohorts enrolled a total of more than $360 \mathrm{HCC}$ cases, and the related gene expression and mutation information are available. In this study, we first performed the statistical analysis, random forest, decision tree, and principal component analysis to identify the differential gene expression, CNV, SNV and SNP profiles, which are associated with the different pathologic stages of TCGA HCC cases. We also analyzed the expression levels of some targeting genes in a Chinese HLivH060PG02 HCC cohort.

\section{Results}

\section{Different pathological grades of HCC in the TCGA cohort}

We obtained the expression and clinical data of 367 hepatocellular carcinomas, 3 fibrolamellar carcinomas, 7 hepatobiliary mixed carcinomas, and 50 adjacent normal controls from the TCGA-LIHC project (Fig. 1A). We first investigated the association between the histological grades of HCC (Fig. 1B, G1, G2, G3, and G4) and clinical outcomes of liver cancer. As shown in Fig. 1C, there was no statistically significant difference in overall survival (OS) and disease-free survival (DFS) between different histological grades ( $P$ value for Log-rank analysis $>0.05$ ). Figure 1D specifically shows the clinical pathological stages (stage I, II, III-IV) and TNM staging of HCC cases. As expected, stage III-IV or T4 patients had the worst prognosis, whereas stage I or T1 patients had a better prognosis (Fig. 1E, $\mathrm{P}<$ 0.001). Therefore, our study focused on HCC cases.

We analyzed the correlation between different pathological stage (stage I, II, III-IV) and clinical indicators. As shown in Figure S1A-F, the total bilirubin, albumin, fetoprotein, and platelet count indicators, but not creatine and protherombin time, showed a statistically significant association with the different HCC pathological grades. In addition, we did not observe a correlation between HCC pathological grades and other factors, including age, height, weight, race, cluster, and gender (Figure S1G-L). Our study aims at analyzing the gene expression and mutational profiles associated with different clinical pathological grades of HCC in the TCGA cohort.

\section{Differential gene screening}

First, we attempted to screen the genes that exhibit the increment or decrement trend in the normal, stage I, stage II, and stage III-IV groups. A range of differential genes between three comparison groups, including Tumor vs. Normal, stage II vs. stage III, stage III+IV vs. stage II, were identified, using the "EdgeR" package. Figure S2A presents the volcano plots for the above three sets. Then, we performed the intersection analysis of the up-regulated and down-regulated genes. As shown in Figure S2B-C, twelve upregulated genes were screened, but no down-regulated genes were obtained. The twelve up-regulated 
genes did not show the protein interaction relationship (Figure S2D) and mainly exist in stage III+IV, but not with a high proportion (Figure S2D). Figure S2E shows the full name information of these genes.

Next, based on the GEPIA online database, we analyzed the expression levels of these genes in normal and tumor, and different pathological grades of HCC. As shown in Figure S3A-B, except for the CRTAC1 gene, other genes were highly expressed in the tumor group, compared with the normal control group. However, only the gene expressions of DUCX2, IQCA1, PCSK1, HOXB9, KCNH2, and NPTX1 were statistically correlated with the stage I-IV distribution. The results of the overall survival and disease-free survival analysis further indicated that the high expression levels of CUZD1 and IQCA1 were associated with poor prognosis of HCC (Figure S3C).

\section{Copy number variation analysis}

We performed the somatic copy number variation (CNV) profile and identified a total of 16,644 genes with CNV from the TCGA HCC dataset. And the Circos 2D track plot for the CNV distribution in the chromosomes was shown in Figure 2A. Then, we utilized the Kolmogorov-Smirnov test to analyze the correlation between CNV and gene expression and obtained a series of genes. After the GO and KEGG analysis, we found that most of these genes were involved in the cell division or cell cycle processes, such as organelle fission, nuclear division, and spindle location (Figure 2B-E). For instance, cell cycleassociated CCNE2 gene in the Tumor, stage I, and stage II group exhibits the single deletion (sd) and single gain (sg) mutations, which are correlated with the gene expression of CCNE2 protein. However, the GADD45G expression level in HCC cases is higher than the negative controls, hinting the presence of other potential gene expression inhibition mechanisms (Figure $2 \mathrm{G}$ ). Figure S4 shows some CNV-driven genes involved in the cell cycle pathway.

\section{Protein-protein interaction network analysis}

Based on the above identified genes, we constructed a protein-protein interaction (PPI) network using the "STRINGdb" package and "Cytoscape" software. We also performed the "Molecular Complex Detection" (MCODE) modular analysis to screen some hub genes within the PPI network. Figure 3A-B shows the two modules with the highest ratings. We further found that the expression levels of these hub genes within the two modules were statistically correlated with CNV. And the cell cycle-related genes, such as TTK, CDC20 and ASPM, were highly expressed and exhibited a significant positive correlation with CNV (Figure 3C).

\section{Genetic mutation analysis}

We downloaded the HCC-related simple nucleotide variation data from the TCGA database, and selected the top 15 genes with the most frequent mutation frequency, such as TTN, TP53, CTNNB1, MUC16, and 
$A L B$, to map the waterfall with clinical grading information. As shown in Figure S7A, the mutation types of these genes are mainly non-synonymous mutations; however, the gene mutation frequency in $286 \mathrm{HCC}$ patients with mutations is not associated with the clinical pathology of HCC (stage I, II, III-IV). In addition, the high expression of CTNNB1 protein was related to the CTNNB1 mutation in overall HCC, stage I, II, IIIIV groups (Figure S7B). TP53 gene mutation was associated with the reduced expression of TP53 in the overall HCC, stage I, II groups (Figure S7B). The OBSCN mutation also correlated with the low expression of $O B S C N$ in overall HCC and specific stage I groups (Figure S7B).

We further conducted the waterfall map analysis on the above-mentioned CENPF, ASPM, MELK, TTK, GADD45G, CDC20, CCNE2, and other interesting genes, and did not observe the correlation between the low mutation frequency of these genes and pathological stages or gene expression, although mainly nonsynonymous mutations as well (Figure S6). In addition, variations in the CTNNB1, TP53, TTN, and OBSCN genes were not found to be linked to the clinical prognosis in different pathological grades (Figure S7-8).

Next, we extracted the SNP data of HCC from the TCGA cohort and found that the rs121913396, rs121913400, rs121913407 SNP of CTNNB1 and rs28934571 SNP of TP53 gene were relatively high frequency (Figure S9A). There are more than ten types of SNP for CTNNB1 gene (Figure S9B). Compared with the wild type group, the CTNNB1 gene with rs121913396 and rs121913400 showed a higher expression level (Figure S9C). Nevertheless, we did not observe the positive correlation between the rs121913396, rs121913400, rs121913407 of CTNNB1 gene, and HCC clinical prognosis (Figure S10A-C). Although we did not detect the relationship between TP53 rs28934571 and gene expression (Figure S9C), the prognosis of AA and CA genotypes of TP53 rs28934571 was poorer than that of wild type (Figure S10D).

\section{Random forest and decision tree analysis}

We combined the above clinical, mutation and expression information to perform the random forest modeling analysis. Multiple dimension scale plot in Figure S11A suggested the effective classification of negative normal and overall HCC group. AUC value of ROC equals to 0.956 , indicating high classification accuracy (Figure S11B). We also showed the feature vectors extracted from the classification model in Figure S11C-D, and obtained the largely contributed genes, such as ECM1, FCN2, ANGPTL6, OIT3, ADAMTS13 and LRRC14. Next, we performed the decision tree modeling analysis, based on the above genes. We first randomly selected $260 \mathrm{HCC}$ cases for modeling, and then test other 125 cases, and finally found that the predicted rate of the genes was larger than $90 \%$ (Figure S11E). Meanwhile, we compared the expression of these genes in $50 \mathrm{HCC}$ patients with adjacent non-tumor and found that ECM1, FCN2, ANGPTL6, OIT3 genes in overall HCC tissue showed the higher expression level than the adjacent nontumor tissue (Figure S11F, $P<0.0001$ ).

Subsequently, we performed random forest modeling with different pathological stages, which is primarily based on TNM information. To prove the validity of this classification method, we performed random forest and decision tree modeling without removing TNM information and found that T1 and T2 
information can effectively distinguish stage I, II, III-IV with the AUC value of 0.994 in ROC (Figure 4A-C). Then, we removed the TNM information for random forest modeling and found that the classification effect was reduced (Figure 4D-E, AUC=0.675). Figure 4F-G shows the genes that contribute significantly to the classification model. We then performed the decision tree analysis with 210 cases for training and 116 cases for testing. The result showed a prediction accuracy of $56.0 \%$ (Figure $4 \mathrm{H}$ ). Compared with 50 normal adjacent controls, VIPR1, FAM99A, and GNA14 genes were down-regulated in 50 HCC tissues (Figure $4 \mathrm{l}, P<0.0001)$, while CEP55, SEMA3F, and PRR11 genes were highly expressed $(P<0.0001)$. The expression of these genes is closely related to different pathological stages (Figure $4 \mathrm{~J}$ ).

\section{Principal component analysis}

Finally, we used principal component analysis (PCA) to screen the target genes associated with different pathological grades of HCC. As shown in Figure $5 \mathrm{~A}$, the calculated variances of the principal component (PC) 1 , 2, and 3 equaled to $9.4 \%, 8.1 \%$, and $6.3 \%$, respectively. Based on the PC1/2 (Figure 5B) and $\mathrm{PC} 1 / 2 / 3$ (Figure $5 C$ ), we can effectively classificate the negative normal and overall HCC groups, but not the stage I, II, III-IV groups. Figure 5D shows the top 10 genes that contributing mainly to PC1 and PC2. We analyzed the expression level of these genes between HCC tissue and adjacent normal tissue, or in different pathological stages. As shown in Figure 5E-F, compared with normal tissue, the SLC27A5, $A L D H 2$, and DCXR genes were down-regulated $(P<0.0001)$, while LAMTOR4 $(P=0.003)$, SNRPA $(P$ $<0.0001)$ SNRPD2 $(P<0.0001)$ genes were highly expressed, in overall HCC tissues. In addition, the expression of the SLC27A5, ADAM17, SNRPA, SNRPD2, and ALDH2 genes was associated with different pathologic stages (Figure $5 \mathrm{E}-\mathrm{F}$ ).

\section{HLivH060PG02 HCC cohort analysis}

After the above analyses of TCGA cohort, we obtained a series of HCC pathological grade-associated genes. We further assessed the survival prognosis value and of these genes through GEO database (data not shown), and analyzed the research status of genes through the on-line PubMed database retrieval. Thus, seven interesting genes, including GAS2L3, CUZD1, SNRPA, SNPRD2, SEMA3F, IQCA1, OIT3, were screened out. We analyzed the expression difference of these genes between the HCC tissues and corresponding adjacent normal tissues, in the Chinese HLivH060PG02 HCC cohort. Unfortunately, due to the lower amplification efficiency of the IQCA1 and OIT3, we finally analyzed the remaining five genes, namely GAS2L3, CUZD1, SNRPA, SNPRD2, and SEMA3F. Figure 6A illustrates the correlation between GAS2L3, SNRPA, SNRPD2 and the prognosis of HCC, as example. As shown in Figure 6B, compared with adjacent normal tissues, we observed a highly expressed level of $G A S 2 L 3(P=0.036), \operatorname{SNRPA}(P<0.001)$, and SNRPD2 $(P=0.002)$ genes in HCC tissues. Moreover, these three genes in pathologic stage III showed a higher expression trend then that in stage III, but statistical significance was only detected for the GAS2L3 gene $(P=0.013)$. Considering the small sample size, we do not rule out the correlation between SNRPA, SNRPD2 genes and pathological stage of HCC. 


\section{Discussion}

Considering the complexity of etiology and pathogenesis of liver cancer, it is essential to continuously screen and analyze the target genes closely related to the pathogenesis of liver cancer. Based on the expression, mutation and clinical data of liver cancer cases in the TCGA cohort, we aim at identifying the potential liver cancer-associated targeting genes. It should be noted that the TCGA-LIHC project includes not only hepatocellular carcinomas (HCC) cases but also a small amount of fibrolamellar carcinomas and hepatobiliary mixed carcinomas cases. Taking account of the differences of distinct liver cancer types and the factor of sample size, we only selected the cases of HCC, the most common primary liver malignancy. The current reports regarding HCC-related target genes from the different clinical pathological stages (stage I, II, III, IV)or histological grades of (G1, G2, G3 and G4)HCC cases in TCGA cohort are very limited, even though several publications from other aspects or with different analysis strategies were retrieved [8-10]. We did not observe a correlation between histological grades of $\mathrm{HCC}$ and clinical outcomes through the survival curve analyses. As expected, different clinical pathological stages of $\mathrm{HCC}$ are closely related to clinical prognosis. Therefore, we attempted to screen the potential target genes associated with normal, stage I, II, III-IV pathological classification of HCC.

The alteration of the target gene expression level leads to the abnormality of the corresponding protein function, which is the critical mechanism underling the hepatocarcinogenesis [11-13]. Based on the sample size, we combined the data of stage III and IV, and focused on the differentially expressed genes associated with normal, stage I, stage II, and stage III-IV classifications. We tried toutilize the "EdgeR" package for the statistically significant differential genes in three comparisons (Tumor vs. Normal, stage II vs. stage III, stage III + IV vs. stage II), and further screen the intersection gene. Through this strategy, we did not identify the target genes with a decreasing trend in the groups of normal, stage I, stage II and stage III + IV), but several genes with increasing trend (e.g.DUCX2, IQCA1, PCSK1, etc.), which showed the low expression frequency and mainly gathered in stage III-IV. This analysis strategy is not effective. Subsequently, we used the Principal Component Analysis (PCA) approach [14, 15]to decrease the dimensionality of the datasets for the group of normal, stage I, stage II, and stage III-IV, and to screen for the genes that contributed largely to the main component. It was found that the normal and tumor group can be better distinguished by the principal components of 1,2 , and 3 , but not the groups of stage I, stage $\mathrm{II}$, and stage III-IV, may due to the low sample size and the complexity of different pathological staging mechanisms of HCC. Despite this, we also obtained the top 10 genes that contributed mostly to the principal component 1 and 2. Of them, the expression levels of the SLC27A5, SNRPA, SNRPD2, and ALDH2 genes were significantly associated with different pathological stages of HCC. Up to now, only one study reported that DNA hypermethylation could reduce the expression of SLC27A5 in HCC, which contributing to HCC progression through NRF2/TXNRD1 pathway [16]. Two studies based on the mouse model indicated the potential role of $A L D H 2$ expression in the hepatocellular carcinogenesis $[17,18]$. Our Chinese HLivH060PG02 HCC cohort analysis first provided the potential role of GAS2L3 and two U1 snRNP component genes (SNRPA and SNRPD2)[19] in the HCC carcinogenesis, and there are still no relevant systematic reports. Therefore, it is meaningful to further explore the molecular mechanism of these genes in the progression and prognosis of HCC. 
Genetic copy number variation (CNV) is caused by the genome rearrangement-induced the copy number amplification or deletion of a large genome fragment $(>1 \mathrm{~kb})$ [20-22]. CNV-induced the change of gene expression level is an essential mechanism of tumorigenesis [23-25]. Even though one relevant study of liver cancer was reported [26],we utilized the distinct analysis strategy to screen out the interesting genes with CNV that are related to gene expression and clinical pathological stages of HCC in TCGA cohort. Our results identified a group of cell cycle or cell division-associated genes, such as GADD45G and CCNE2, exhibited the CNV-driven gene expression. In addition, we utilized MCODE modular analysis to screen out some key genes, such as TTK and CDC20, from the perspective of protein binding, which are also related to cell cycle and division behavior. It is worth noting that the expression levels of some down-regulated genes in HCC (e.g., GADD45G, FPR2, PPBP, etc.) are closely linked to the CNV in a dose dependent manner. Some other key inhibition mechanisms of gene expression, such as hypermethylation modification, may exist for these genes, apart from CNV.

Genetic mutations are considered as the key mechanisms of tumorigenesis[27, 28], and single nucleotide polymorphisms are closely related to the susceptibility of tumors in the population [29]. We performed a series of gene mutation analysis as well. We found that the major mutation types of these genes are nonsynonymous mutations, and the gene mutation frequency is not associated with the clinical pathology of HCC. Even though there is a correlation between the overall variation and expression of CTNNB1 and TP53 genes in HCC and different pathological stages, no positive results were obtained for the mutations of specific site in the relatively low amount of cases. In addition, there are more than 10 SNPs for the CTNNB1 gene in HCC, but with low frequency. We also did not observe the correlation between these SNPs and CTNNB1 high expression or clinical prognosis of HCC. More HCC cases may be required for the confirmation of this point.

We integrated the expression data of all target genes obtained from the above methods, somaticmutation data, and some clinical biochemical indicators, and then utilized the random forest, a robust classification and regression approach [30],for the classification analysis of normal, tumor and stage I, stage II, stage III-IV groups. Although the classification effect of stage I, II, III-IV is worse than the normal/tumor, we identified some key contributing genes (e.g., VIPR1, FAM99A, GNA14CEP55, SEMA3F, $P R R 11$, etc.), which are closely related to different clinical stages. Interestingly, the interest genes screened by the random forest approach (e.g., ECM1, FCN2, ANGPTL6, OIT3, ADAMTS13, etc.) are mainly down-regulated in HCC, compared with the normal group.

\section{Conclusion}

In summary, based on the data of HCC cases in TCGA cohorts, we first conducted the statistical analysis, random forest, decision tree and principal component analysis to identify the differential gene expression, CNV, SNV and SNP profiles, which are associated with the different pathologic stage I, II, and III-IV. More molecular biology experiment so rclinical sample tests are required to further investigate whether the identified genes serve as the prognostic biomarker or therapeutic targets of HCC. 


\section{Materials And Methods}

\section{HCC pathological stage-related gene expression analysis}

We utilized the "TCGAbiolinks" R package to download the liver cancer-associated mRNA, IncRNA expression data with the workflow type of "HTSeq-Counts" and clinical data from the TCGA-LIHC project within the TCGA database (http://tcga-data.nci.nih.gov/tcga/). We extracted the clinical information, including gender, age, race, ethnicity, height, weight, clinical pathologic stage, pathologic T/N/M stage, neoplasm histologic grade, survival status, follow-up time and various clinical, biochemical indicators. There groups of clinical pathologic stages, namely stage I, II and III-IV, were analyzed. Combined with other clinical indicators, we performed the Kruskal-Wallis test or chi-square test, using GraphPad Prism 5.1 software. We also conducted the log-rank test and Kaplan-Meier (KM) survival curve analysis using SPSS 20.0 statistical analysis software.

Based on the R language software (https://www.r-project.org/), we combined the expression data and clinical data, and removed the non-HCC case data. An "EdgeR" package was then utilized for TMM data standardization and differential gene screening. The gene expression level was processed into logarithm base 2 ( $\log 2)$. We obtained the Volcano maps through a "ggplot" package. We also utilized the online venn tool (http://bioinformatics.psb. ugent.be/ webtools/Venn/) for intersection analysis to obtain the intersection genes of different groups, and then used the Morpheus online software [https://software.broadinstitute.org/ Morpheus/] to draw a heat map of cluster analysis. ID conversion is implemented based on the gene ID conversion tool of DAVID (https://david.ncifcrf.gov/conversion.jsp). Protein-protein interaction network analysis of intersection genes was performed based on the STRING online analysis tool (https://string-db.org/). The survival curves and the expression status of specific genes in the groups of total HCC, control, stage I, stage II and stage III-IV were analyzed through the GEPIA, a web server for cancer and normal gene expression profiling and interactive analyses.

\section{Copy number variation analysis}

We first downloaded the copy number variation (CNV) data of the TCGA-LIHC project from the TCGA database page with the type of masked copy number segment, and then used the Perl script to add the according gene annotation based on the CNV chromosome location information. Segment_mean value between -0.2 and +0.2 will be considered as no variation and will be marked as " 0 ". CNV contains the double deletion ( $\mathrm{dd}$, " -2 "), single deletion (sd, "-1"), single gain ( $\mathrm{sg}$, " +1 "), and amplication $\left(\mathrm{A},{ }^{\prime \prime}+2\right.$ or $+>2$ ")] of gene copy number. The chi-square test and the Bonferroni-adjusted $P$ value correction method were utilized to obtain the CNV differential targeting genes between HCC and the normal control group, and then the "RCircos" package was used to obtain the Circos 2D track plot.

Also, we combined gene expression data with CNV differential targeting gene data, and performed Kolmogorov-Smirnov test for correlation analysis to identify the expression-correlated targeting genes with CNV. Then, the enrichGO () function was used for the Gene Ontology (GO) analysis, while 
enrichKEGG () function was for the Kyoto Encyclopedia of Genes and Genomes (KEGG) pathway enrichment analysis. We used the "STRINGdb" package to build a protein interaction network and visualized the results using Cytoscape software. Based on the "Molecular Complex Detection" (MCODE) modular analysis, we screened key hub genes in the PPI network using default settings.

\section{Random forest and decision tree analysis}

Upon the combination of the above clinical, mutation, and expression information, we used the "random Forest" package to perform the random forest modeling analysis. The specific gene profiles of normal, overall HCC and HCC with different clinical pathological grades were effectively classified by the principles of mean decrease accuracy and mean decrease Gini, and visualized by a "ggpubr" package. MDSplot () function was used to obtain the Multi Dimension Scale. Using the "pROC" package, the receiver operating characteristic (ROC) curve is plotted, and the area under the ROC curve (AUC) value is calculated. Based on the results of random forest analysis, the Decision tree modeling analysis is performed, using "rpart" and "rpart.plot" packages.

\section{Genetic mutation analysis}

We directly downloaded the simple nucleotide variation (SNV) data of the TCGA-LIHC project with the type of masked somatic mutation, and extracted the mutation data using the Perl script. According to the mutation rate, the top 15 genes were selected, and the "GenvisR" package was used to draw a waterfall map with clinical grading information. We also extracted the single nucleotide polymorphism (SNP) data, and performed the wilcox test and boxplot () function for the correlation analysis of gene mutation and expression in overall HCC and different pathological stages. The "survminer" package was further used to correlate the specific gene mutations in overall HCC and different pathological stages with the clinical prognosis, and drew the corresponding survival curves.

\section{Principal component analysis}

Based on the gene expression matrix, we used the prcomp () function for the principal component analysis (PCA) to screen the genes associated with different pathological stages of HCC. The "factoextra" and "ggplot2" packages were utilized to obtain the principal component (PC) gravity and gene contribution maps. A three-dimensional map (PC1, PC2, and PC3) is drawn using a "scatterplot3d" package; while a two-dimensional map (PC1, PC2) is obtained by a "ggord" package. In addition, for specific genes selected by a decision tree, random forest, and principal component analysis, we utilized the R language to obtain the expression data of overall HCC tissue and adjacent normal tissue, and perform the t test using GraphPad Prism 5.1 software. We also obtained the expression data of stage I, II, III and IV through the GEPIA. 


\section{HLivH060PG02 HCC cohort analysis}

Based on the enrolled HCC patients in the Chinese population, namely HLivH060PG02 cohort (Shanghai Outdo Biotech Co., Ltd, Shanghai, China), we analyzed the expression levels of five targeting genes (GAS2L3, CUZD1, SNRPA, SNPRD2, SEMA3F), and their correlation with pathologic stages. The clinical characteristics of HCC cases were shown in Table S1, and the use of human biological materials (Number: YB M-05-02) was approved by the Use Ethics Committee of Shanghai Outdo Biotech Company. RNA samples were extracted from a total of $30 \mathrm{HCC}$ tissues and 30 corresponding adjacent normal tissues, respectively. Bases on the synthesized cDNA, a quantitative real-time PCR (qPCR) assay was performed with a TB Green ${ }^{\text {TM }}$ Premix Ex Taq ${ }^{\text {TM }}$ II (Takara, RR820A), using an ABI 7500 Real-Time PCR System (Thermo Fisher Scientific).

The primer sequence information is listed: GAS2L3: 5'-CTGAGGACCCTCCTTGTAGTTG-3' (Forward), 5'CCTTGAAGAGTATCCCAGCCTC-3' (Reverse); CUZD1: 5'-CCAGCCTTTCAACAGTGTGC-3' (Forward), 5'GCCACGAGGTAGCATTTCCT-3' (Reverse); SNRPA: 5'-ACCCGCCCTAACCACACTAT-3' (Forward), 5'GGAGAAGATGGCGTACAGGG-3' (Reverse); SNPRD2: 5'-CAAGTGCTCATCAACTGCCGCA-3' (Forward), 5'GCGGTCTTTGTTGACTGGCTTG-3' (Reverse); SEMA3F: 5'-CAAGGATGTCAACGGCGAGT-3' (Forward), 5'TGAGTCTGGGTCCATGGTGT-3' (Reverse); beta-actin: 5'-GAAGAGCTACGAGCTGCCTGA-3' (Forward), 5'CAGACAGCACTGTGTTGGCG-3' (Reverse). Student's t-test was performed by GraphPad Prism 7.0.4 (San Diego, California USA). Differences with $P<0.05$ were considered significant.

\section{Declarations}

\section{Ethics approval and consent to participate}

The studies involving human participants were reviewed and approved by the clinical research ethics Committee of Shanghai Outdo Biotech Company.

\section{Consent for publication}

Not applicable.

\section{Availability of data and materials}

The datasets generated and/or analysed during the current study are available in the The Cancer Genome Atlas (TCGA) database.

\section{Competing interests}

The authors declare that they have no competing interests. 


\section{Funding}

This work was supported by grants from the Innovation Team Development Plan of the Ministry of Education (IRT13085 to JY), National Nature Science Foundation of China (31670759 to JY, 31571380 to XG, 81572882 to ZY, 31701182 to CS), Excellent Talent Project of Tianjin Medical University (to JY), Tianjin Enterprise Science and Technology Commissioner Project (18JCTPJC59400 to XG).

\section{Author Contributions}

Conceptualization, XJG and JY; Methodology, XJG, CYZ and SYW; Software, XJG and XYC; Validation, YYR and CS; Formal Analysis, XJG and CYZ; Data Curation, XJG, NZ and ZY; Writing-Original Draft Preparation, XJG and CYZ; Writing-Review \& Editing, XJG and JY; Supervision, JY.

\section{Acknowledgements}

Not applicable.

\section{References}

1. Gibbs P, Tie J, Bester L: Radioembolization for hepatocellular carcinoma: current role and future directions - the medical oncologist's perspective. Hepat Oncol 2015, 2(2):117-132.

2. Hartke J, Johnson M, Ghabril M: The diagnosis and treatment of hepatocellular carcinoma. Semin Diagn Pathol 2017, 34(2):153-159.

3. El Jabbour T, Lagana SM, Lee H: Update on hepatocellular carcinoma: Pathologists' review. World J Gastroenterol 2019, 25(14):1653-1665.

4. Raza A, Sood GK: Hepatocellular carcinoma review: current treatment, and evidence-based medicine. World J Gastroenterol 2014, 20(15):4115-4127.

5. Gomaa Al, Waked I: Recent advances in multidisciplinary management of hepatocellular carcinoma. World J Hepatol 2015, 7(4):673-687.

6. Wallace MC, Preen D, Jeffrey GP, Adams LA: The evolving epidemiology of hepatocellular carcinoma: a global perspective. Expert Rev Gastroenterol Hepatol 2015, 9(6):765-779.

7. Wang Z, Jensen MA, Zenklusen JC: A Practical Guide to The Cancer Genome Atlas (TCGA). Methods Mol Biol 2016, 1418:111-141.

8. Xu B, Lv W, Li X, Zhang L, Lin J: Prognostic genes of hepatocellular carcinoma based on gene coexpression network analysis. J Cell Biochem 2019.

9. Wu P, Xiao Y, Guo T, Wang Y, Liao S, Chen L, Liu Z: Identifying miRNA-mRNA Pairs and Novel miRNAs from Hepatocelluar Carcinoma miRNomes and TCGA Database. J Cancer 2019, 10(11):2552-2559. 
10. Agarwal R, Narayan J, Bhattacharyya A, Saraswat M, Tomar AK: Gene expression profiling, pathway analysis and subtype classification reveal molecular heterogeneity in hepatocellular carcinoma and suggest subtype specific therapeutic targets. Cancer Genet 2017, 216-217:37-51.

11. Cai J, Li B, Zhu Y, Fang X, Zhu M, Wang M, Liu S, Jiang X, Zheng J, Zhang X et al: Prognostic Biomarker Identification Through Integrating the Gene Signatures of Hepatocellular Carcinoma Properties. EBioMedicine 2017, 19:18-30.

12. Shangguan $H$, Tan SY, Zhang JR: Bioinformatics analysis of gene expression profiles in hepatocellular carcinoma. Eur Rev Med Pharmacol Sci 2015, 19(11):2054-2061.

13. Pinato DJ, Pirisi M, Maslen L, Sharma R: Tissue biomarkers of prognostic significance in hepatocellular carcinoma. Adv Anat Pathol 2014, 21(4):270-284.

14. Marziali G, Buccarelli M, Giuliani A, llari R, Grande S, Palma A, D'Alessandris QG, Martini M, Biffoni M, Pallini $\mathrm{R}$ et al: A three-microRNA signature identifies two subtypes of glioblastoma patients with different clinical outcomes. Mol Oncol 2017, 11(9):1115-1129.

15. Jolliffe IT, Cadima J: Principal component analysis: a review and recent developments. Philos Trans A Math Phys Eng Sci 2016, 374(2065):20150202.

16. Gao Q, Zhang G, Zheng Y, Yang Y, Chen C, Xia J, Liang L, Lei C, Hu Y, Cai X et al: SLC27A5 deficiency activates NRF2/TXNRD1 pathway by increased lipid peroxidation in HCC. Cell Death Differ 2019.

17. Hou G, Chen L, Liu G, Li L, Yang Y, Yan HX, Zhang HL, Tang J, Yang YC, Lin X et al: Aldehyde dehydrogenase-2 (ALDH2) opposes hepatocellular carcinoma progression by regulating AMPactivated protein kinase signaling in mice. Hepatology 2017, 65(5):1628-1644.

18. Seo W, Gao Y, He Y, Sun J, Xu H, Feng D, Hee Park S, Cho YE, Guillot A, Ren T et al: ALDH2 deficiency promotes alcohol-associated liver cancer by activating oncogenic pathways via oxidized DNA enriched extracellular vesicles. J Hepatol 2019.

19. Azam S, Hou S, Zhu B, Wang W, Hao T, Bu X, Khan M, Lei H: Nuclear retention element recruits U1 snRNP components to restrain spliced IncRNAs in the nucleus. RNA Bio/ 2019, 16(8):1001-1009.

20. Zarrei M, MacDonald JR, Merico D, Scherer SW: A copy number variation map of the human genome. Nat Rev Genet 2015, 16(3):172-183.

21. Nowakowska B: Clinical interpretation of copy number variants in the human genome. $J$ App/ Genet 2017, 58(4):449-457.

22. Liang L, Fang JY, Xu J: Gastric cancer and gene copy number variation: emerging cancer drivers for targeted therapy. Oncogene 2016, 35(12):1475-1482.

23. Takai A, Dang HT, Wang XW: Identification of drivers from cancer genome diversity in hepatocellular carcinoma. Int J Mol Sci 2014, 15(6):11142-11160.

24. Gu DL, Chen YH, Shih JH, Lin CH, Jou YS, Chen CF: Target genes discovery through copy number alteration analysis in human hepatocellular carcinoma. World J Gastroentero/ 2013, 19(47):88738879 . 
25. Chen Y, Chen C: DNA copy number variation and loss of heterozygosity in relation to recurrence of and survival from head and neck squamous cell carcinoma: a review. Head Neck 2008, 30(10):13611383.

26. Lu X, Ye K, Zou K, Chen J: Identification of copy number variation-driven genes for liver cancer via bioinformatics analysis. Oncol Rep 2014, 32(5):1845-1852.

27. Zucman-Rossi J, Villanueva A, Nault JC, Llovet JM: Genetic Landscape and Biomarkers of Hepatocellular Carcinoma. Gastroenterology 2015, 149(5):1226-1239.e1224.

28. Schulze K, Nault JC, Villanueva A: Genetic profiling of hepatocellular carcinoma using nextgeneration sequencing. J Hepato/ 2016, 65(5):1031-1042.

29. Koberle B, Koch B, Fischer BM, Hartwig A: Single nucleotide polymorphisms in DNA repair genes and putative cancer risk. Arch Toxicol 2016, 90(10):2369-2388.

30. Liu Y, Zhao H: Variable importance-weighted Random Forests. Quant Bio/ 2017, 5(4):338-351.

\section{Figures}
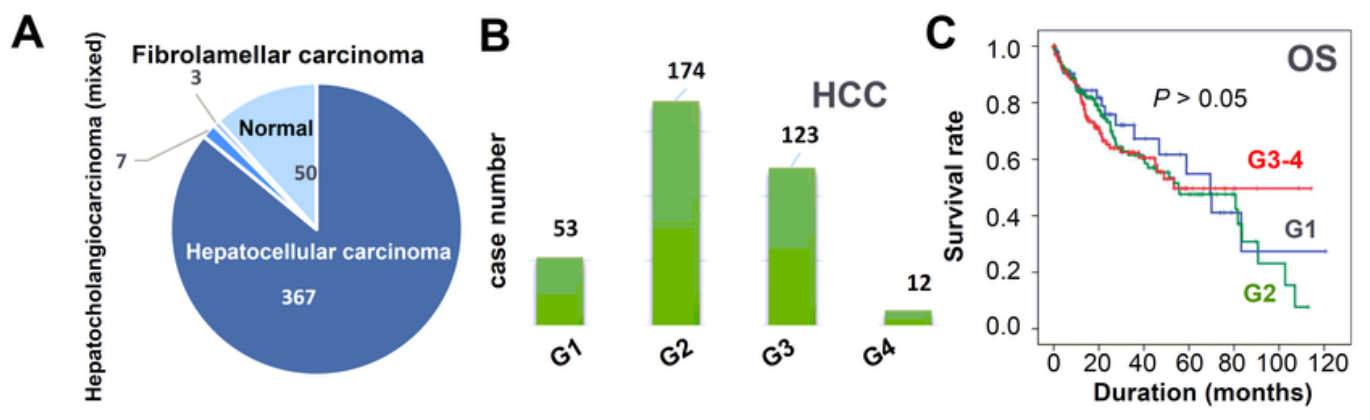

D
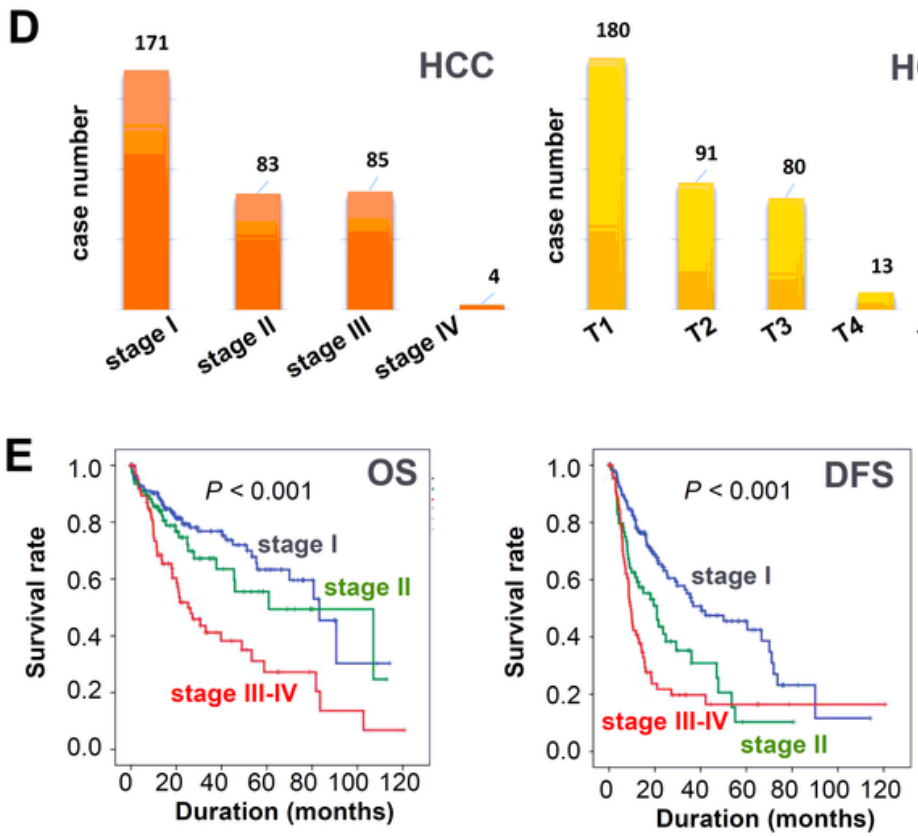
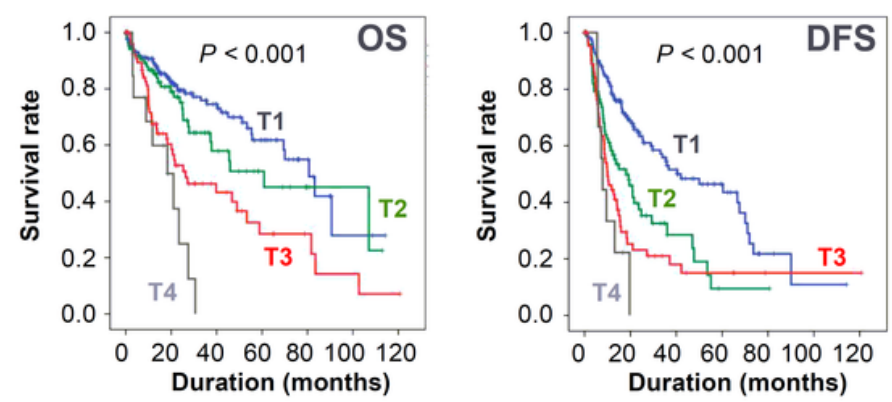
Figure 1

Survival curve analysis for the different pathologic stages of HCC patients in the TCGA database. (A) The included liver cancer cases and adjacent normal controls in TCGA cohorts. (B) The neoplasm histologic grades (G1, G2, G3 and G4) of HCC cases. (C) The log-rank test and KM survival curve analysis according to the histological grades of HCC were performed. (D) The clinical pathologic stages (stage I, stage II and stage III-IV) and T/N/M stage of HCC cases. (E) The survival curve analysis according to the stage I, II, IIIIV and T1-T4 were performed.
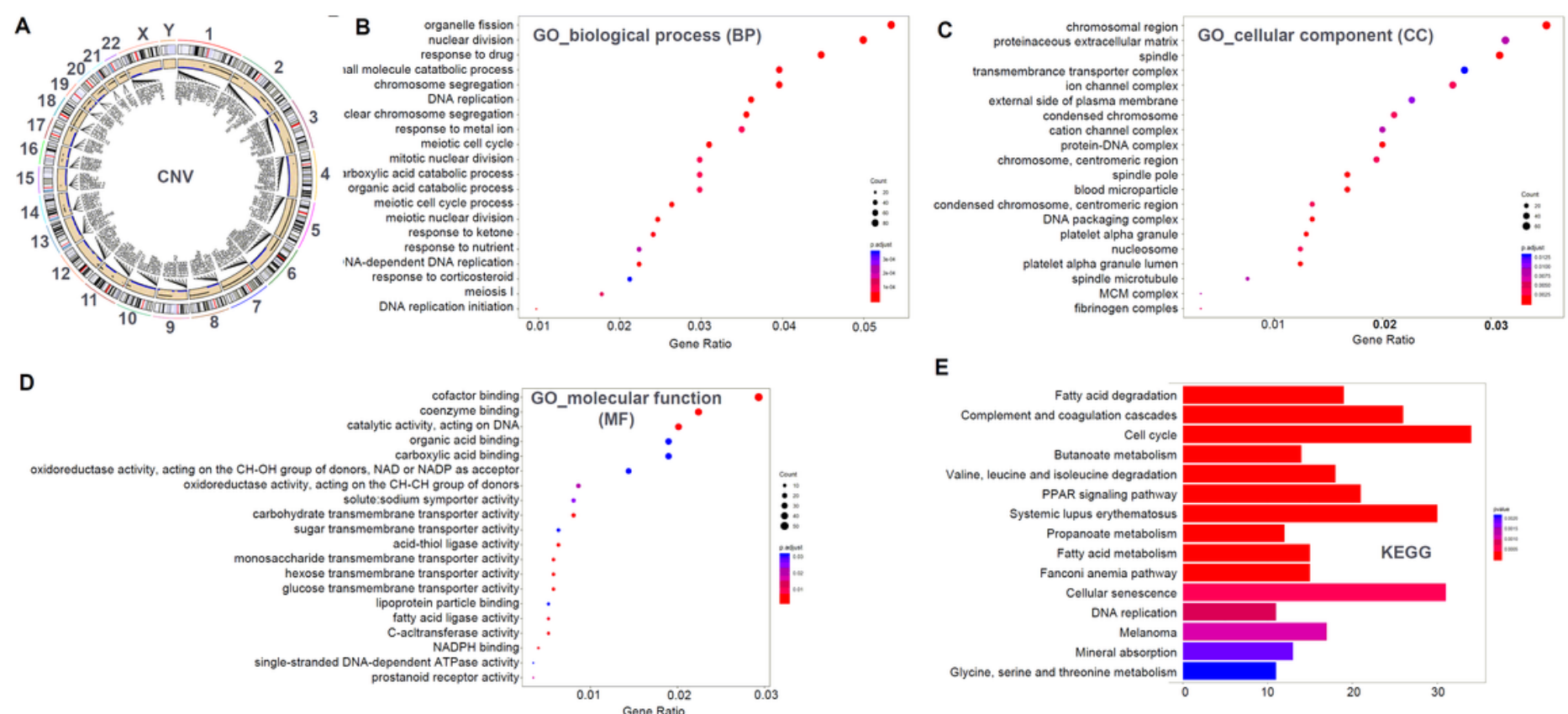

$\mathbf{F}$
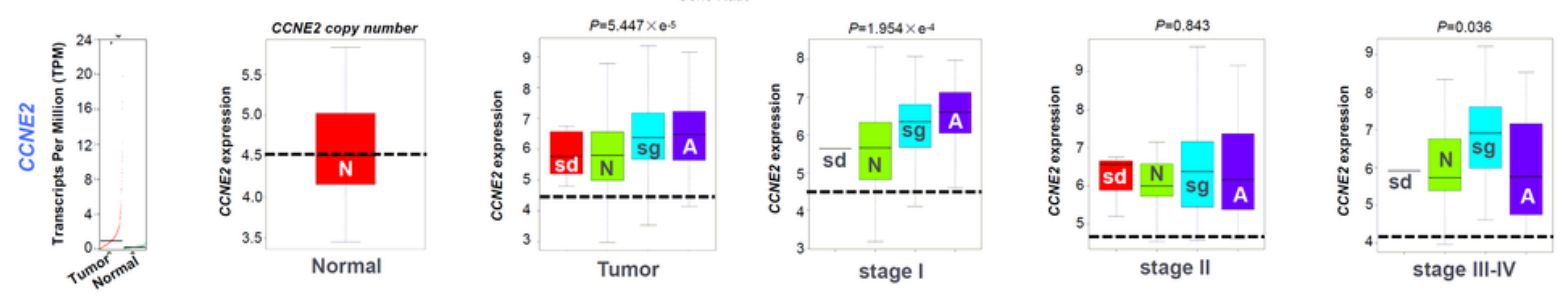

G
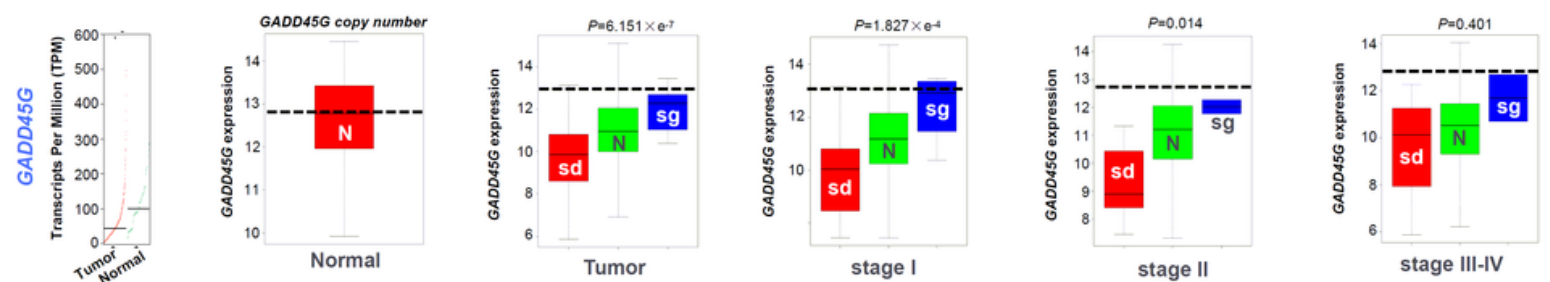

Figure 2

\section{Figure 2}

Genetic copy number variant analysis for the different pathologic stages of HCC patients. (A) Circos 2D track plot of CNV profile was showed. (B-D) GO and KEGG analysis data of the genes with CNV, which was correlated with the gene expression. (E-F) We analyzed the expression levels of CCNE2, GADD45G 
genes in normal and tumor by GEPIA, and the correlation between gene expression and CNV in normal and different pathological grades of HCC.
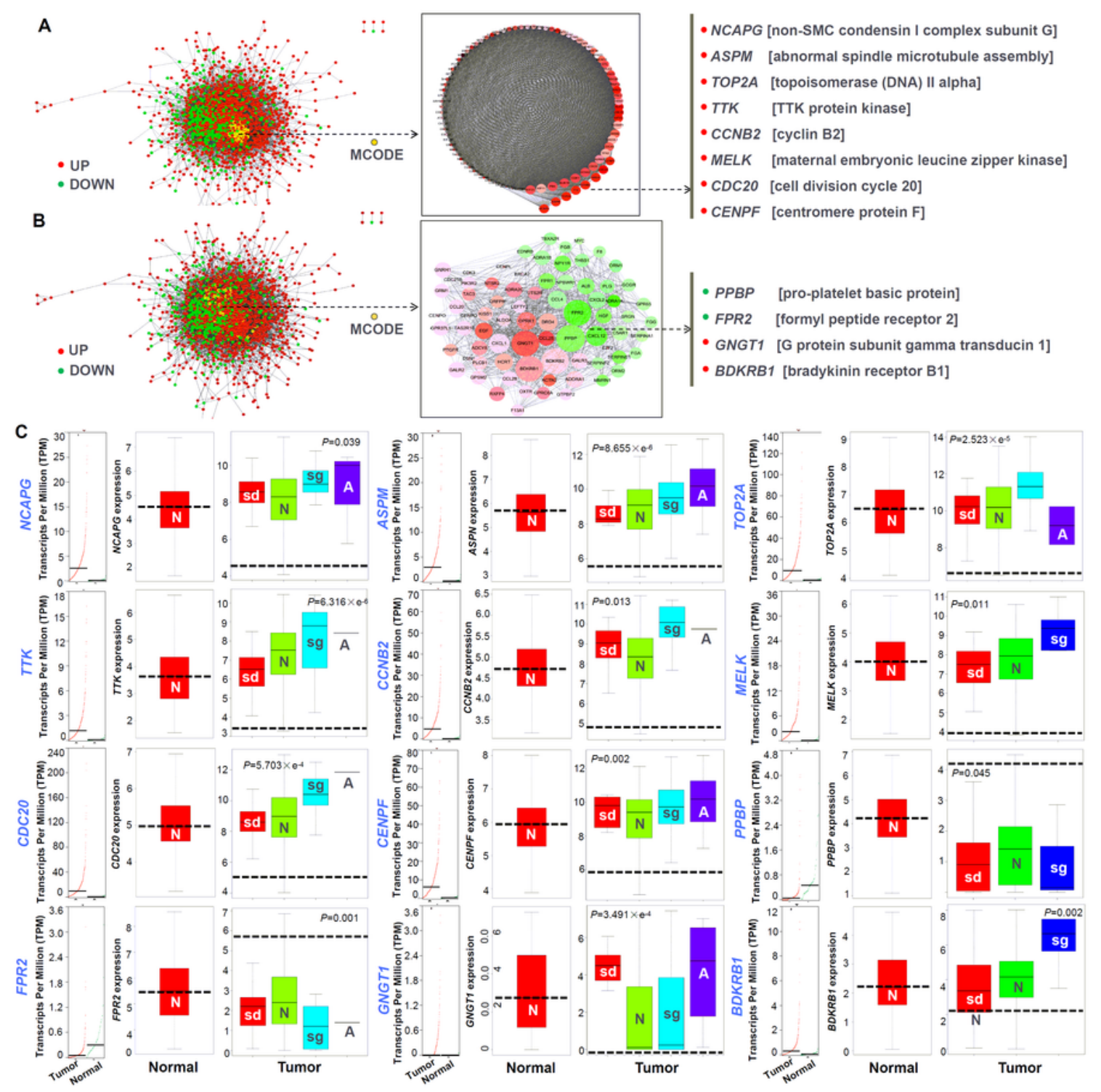

Figure 3

\section{Figure 3}

The protein-protein interaction network. (A-B) "STRINGdb" package and Cytoscape software, and "Molecular Complex Detection" (MCODE) were utilized for the construction of protein-protein interaction (PPI) network and the identification of hub genes. (C) The expression levels in normal and tumor, and the 
correlation between gene expression and CNV of some hub genes in normal and different pathological grades of HCC were analyzed.

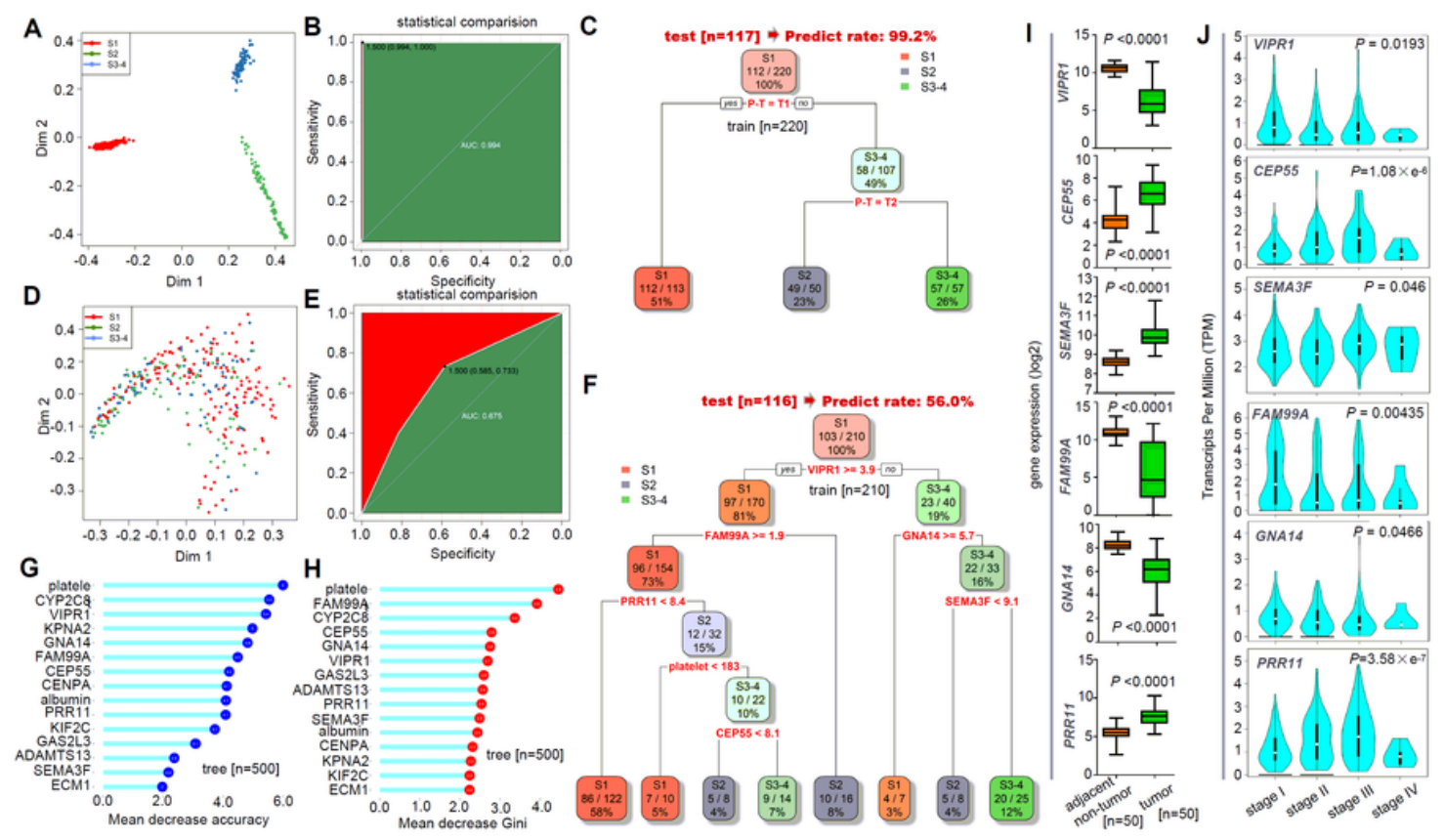

Figure 4

\section{Figure 4}

The decision tree and random forest analysis for the different pathologic stages of HCC patients in the TCGA cohort. (A) We combined the clinical, mutation and expression information to perform the random forest modeling analysis. Multiple dimension scale plot was provided. (B) ROC curve is plotted, and the AUC value is calculated. (C) Decision tree modeling analysis is performed. (D-F) We removed the TNM 
information to complete the random forest modeling, again. (G-H) Based on the principles of mean decrease accuracy and mean decrease Gini, we identified the largely contributed genes. (I-J) We compared the expression of these genes in $50 \mathrm{HCC}$ tissues with adjacent non-tumor tissues and obtained the expression data of stage I, II, III, and IV through the GEPIA.
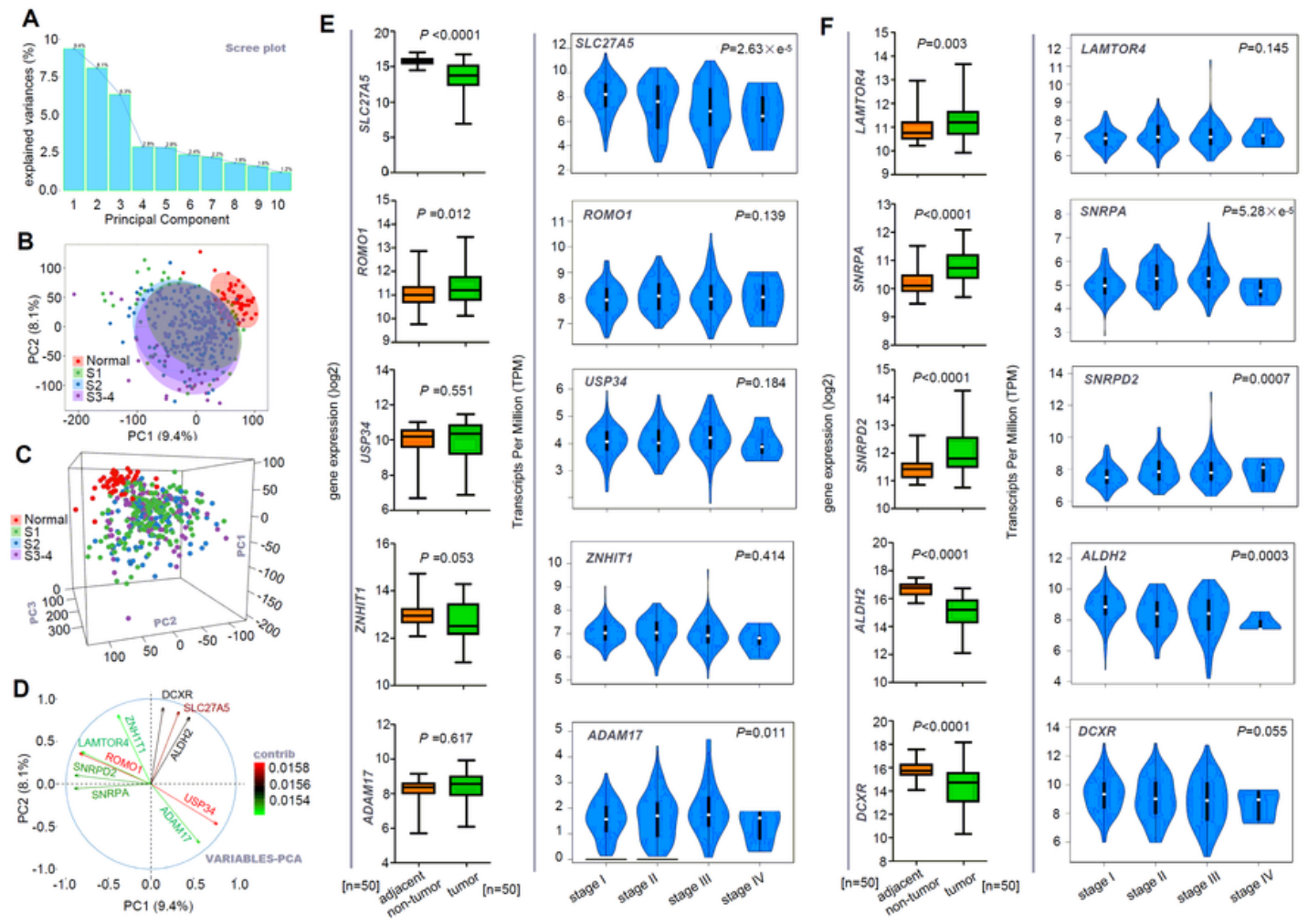

Figure 5

\section{Figure 5}

The PCA analysis for the different pathologic stages of TCGA HCC cases. We performed the principal component analysis (PCA) to screen the genes associated with different pathological stages of HCC. The 
principal component (PC) gravity (A), a two-dimensional map (PC1/PC2) (B), a three-dimensional map (PC1/PC2/PC3) (C) and gene contribution maps (D) were provided. (E-F) We analyzed the expression level of overall HCC tissue, adjacent normal tissue, and stage I, II, III, and IV group.
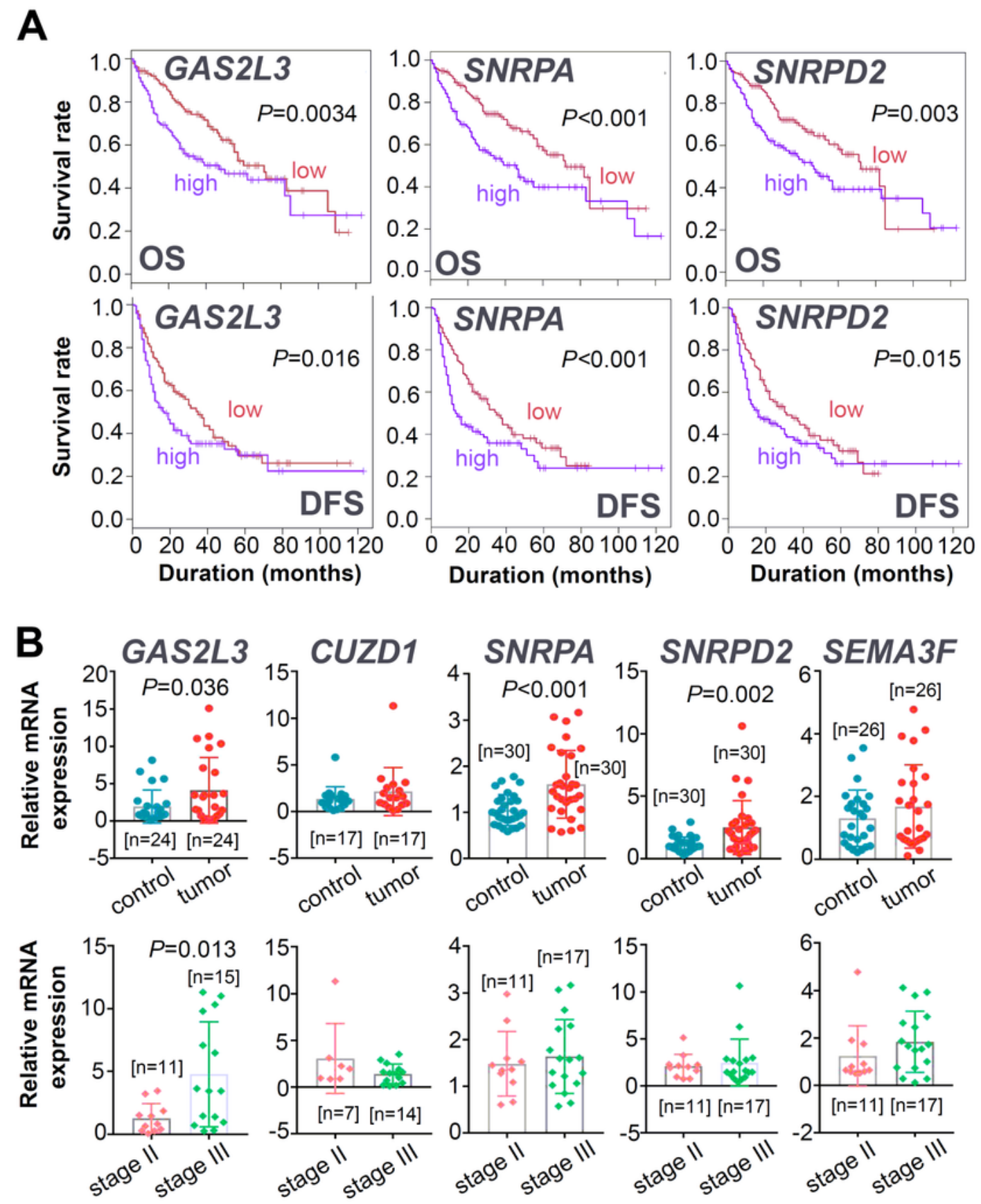

\section{Figure 6}

Figure 6

The expression level of five targeting genes in the HCC cases of Chinese population. (A), For the GAS2L3, SNRPA, and SNPRD2, we performed the Kaplan-Meier estimates of the overall survival (OS) or disease- 
free survival (DFS) through GEPIA, based on the data in TCGA cohorts; (B) We further performed a qPCR assay to detect the expression levels of five targeting genes (GAS2L3, CUZD1, SNRPA, SNPRD2, SEMA3F) in the HLivH060PG02 HCC cohort of Chinese population, and their correlation with pathologic stages of HCC. Student's t-test was performed and significant differences were indicated.

\section{Supplementary Files}

This is a list of supplementary files associated with this preprint. Click to download.

- SUPPLEMENTARYMATERIAL.docx 